\title{
Prévalence de la conformité aux directives en matière d'activité physique pour la prévention du cancer en Alberta
}

\author{
F. E. Aparicio-Ting, Ph. D. (1,2); C. M. Friedenreich, Ph. D. (1,3,4); K. A. Kopciuk, Ph. D. (1,3,5); \\ R. C. Plotnikoff, Ph. D. (6); H. E. Bryant, Ph. D. $(4,7)$
}

Cet article a fait l'objet d'une évaluation par les pairs.

\section{Résumé}

Introduction : Des directives portant sur les niveaux d'activité physique (AP) souhaitables ont été élaborées par la Société canadienne de physiologie de l'exercice (SCPE) et le Department of Health and Human Services des États-Unis (USDHHS) pour l'obtention de bienfaits pour la santé, et par l'American Cancer Society (ACS) et le World Cancer Research Fund/American Institute for Cancer Research (WCRF/AICR) pour l'obtention de bienfaits quant à la prévention du cancer.

Méthodologie : Nous avons évalué si ces directives étaient suivies au moyen d'un échantillon composé de 14294 Albertains de 35 à 64 ans ayant participé au Tomorrow Project et recrutés entre 2001 et 2005 . Nous avons eu recours à une analyse de régression logistique pour examiner les corrélats des comportements en matière d'AP de loisir.

Résultats : Selon les estimations, les participants se conformaient aux directives à hauteur de respectivement $55 \%$ pour la SCPE, $42 \%$ pour l'ACS, $26 \%$ pour l'USDHHS et $23 \%$ pour le WCRF/AICR. Les femmes étaient moins nombreuses que les hommes à se conformer aux directives de l'ACS (rapport des cotes $[\mathrm{RC}]=0,72$, intervalle de confiance $[\mathrm{IC}]$ à $95 \%$ : $0,55$ à 0,93$)$, de l'USDHHS ( $R C=0,67$, IC à $95 \%: 0,50$ à 0,89$)$ et du WCRF/AICR $(\mathrm{RC}=0,63$, IC à $95 \%: 0,47$ à 0,85$)$, et l'obésité était corrélée à la non-conformité aux directives de l'USDHHS (RC = 0,45, IC à $95 \%: 0,32$ à 0,65) et du WCRF/AICR $(\mathrm{RC}=0,79$, IC à $95 \%: 0,63$ à 0,98).

Conclusion : Les Albertains, en particulier les femmes et les personnes obèses, ne sont pas assez actifs pour obtenir des bienfaits quant à la prévention du cancer.

Mots-clés : activité physique, prévention du cancer, santé de la population, mode de vie, comportements liés à la santé, directives

\section{Introduction}

Le cancer demeure la deuxième cause de mortalité et de morbidité au Canada, avec des estimations en 2011 de 177800 cas incidents et de 75000 décès $^{1}$. Le fardeau économique total du cancer a été estimé à environ $9 \%$ du coût total des maladies au Canada $^{2}$. Bien que les traitements et la prévention précoce se soient améliorés au cours des dernières décennies, la prévention du cancer par la modification des facteurs de risque liés à l'environnement et au mode de vie demeure la stratégie à long terme la plus viable pour réduire substantiellement le fardeau du cancer au Canada ${ }^{3}$. Plusieurs facteurs de risque modifiables liés au mode de vie ont été abondamment étudiés, notamment le tabagisme, la consommation d'alcool, l'alimentation, l'exposition au soleil et, plus récemment, l'activité physique $(\mathrm{AP})^{4,5}$.

Les données montrant que l'AP est un important facteur de risque modifiable lié au mode de vie susceptible de réduire le risque de plusieurs cancers continuent de s'accumuler. Le risque de cancer du côlon, du sein et de l'endomètre est réduit de $25 \%$ à $30 \%$ chez les personnes physiquement actives, et des données récentes amènent à penser que l'AP pourrait réduire le risque de cancer de la prostate, de l'ovaire, du poumon et des cancers gastro-intestinaux ${ }^{6-9}$. Les données selon lesquelles l'AP pourrait agir sur l'étiologie du cancer sont maintenant considérées comme assez fortes, constantes et biologiquement plausibles. Plusieurs mécanismes biologiques ont été proposés pour expliquer comment l'AP réduit le risque de cancer, notamment par ses effets sur les concentrations endogènes d'hormones sexuelles et d'hormones métaboliques, sur les facteurs de croissance, sur l'inflammation et sur la résistance à l'insuline, facteurs qui influent tous sur la carcinogenèse $^{10-12}$. De plus, l'AP pourrait réduire le risque de cancer en diminuant

Rattachement des auteurs :

1. Department of Population Health Research, Alberta Health Services-Cancer Care, Calgary, (Alberta)

2. Community Health Sciences, Faculty of Medicine, University of Calgary, Calgary (Alberta), Canada

3. Department of Oncology, Faculty of Medicine, University of Calgary, Calgary (Alberta), Canada

4. Department of Community Health Sciences, Faculty of Medicine, University of Calgary, Calgary (Alberta), Canada

5. Department of Mathematics and Statistics, Faculty of Science, University of Calgary, Calgary (Alberta), Canada

6. School of Education, University of Newcastle, New South Wales, Australie

7. Partenariat canadien contre le cancer, Toronto (Ontario), Canada

Correspondance : Fabiola Aparicio-Ting, Community Health Sciences, Faculty of Medicine, University of Calgary, 3rd Floor, TRW Building, 3280 Hospital Drive N.W., Calgary (Alberta) T2N 4Z6; tél. : 403-220-8124; téléc. : 403-270-7307; courriel : feaparic@ucalgary.ca 
l'obésité et l'adiposité centrale, deux facteurs de risque connus de cancer du côlon, de l'endomètre, du rein et de l'œsophage ainsi que de cancer du sein chez les femmes ménopausées ${ }^{12-14}$. L'excès de poids et l'obésité provoquent un déséquilibre des hormones sexuelles et des hormones métaboliques dans le corps et influent sur la disponibilité d'un certain nombre de facteurs de croissance intervenant dans la résistance à l'insuline et dans les voies de l'inflammation qui initient et promeuvent la carcinogenèse $\mathrm{e}^{14}$. Par conséquent, l'AP peut aussi être utilisée pour la prise en charge du poids dans l'optique de réduire le risque de cancer $^{12-14}$.

Ces données d'envergure selon lesquelles l'AP joue un rôle important dans la prévention du cancer et d'autres maladies chroniques ont amené un certain nombre d'organismes à formuler des recommandations ou des directives en matière d'AP. Le but de ces directives est d'encourager les populations inactives à pratiquer des activités physiques et de définir des objectifs qui pourront servir de cibles personnelles et permettre de mesurer les progrès ${ }^{15}$. Au Canada, la Société canadienne de physiologie de l'exercice (SCPE) a élaboré des directives à l'intention des adultes, des personnes âgées et des enfants ${ }^{16}$. Les directives de 2003 de la SCPE recommandent aux adultes de faire au moins 150 minutes d'AP aérobie d'intensité modérée à élevée chaque semaine. Ces directives précisent aussi que s'adonner à plus d'AP offre davantage de bienfaits pour la santé ${ }^{16}$. Une activité d'intensité modérée est définie comme une activité aérobie qui n'est pas épuisante et qui provoque une légère transpiration (p. ex. la marche rapide), alors que, dans une activité d'intensité élevée, la personne transpire et est essoufflée, et son rythme cardiaque est rapide (p. ex. jogging, danse aérobique) ${ }^{17-18}$. La SCPE recommande aussi aux adultes d'intégrer des activités de renforcement de leurs muscles et de leurs os au moins deux jours par semaine; notre étude vise toutefois uniquement les niveaux d'activité aérobie.

L'American Cancer Society (ACS) ${ }^{19}$, le Department of Health and Human Services des États-Unis (USDHHS) en collaboration avec le Department of Agriculture des États-Unis ${ }^{20}$, et le World
Cancer Research Fund en collaboration avec l'American Institute for Cancer Research (WCRF/AICR) ${ }^{21}$ recommandent aussi au moins 150 minutes d'AP d'intensité modérée à élevée par semaine pour obtenir des bienfaits pour la santé générale. Ils recommandent en outre davantage d'AP pour prévenir d'autres maladies chroniques. Après un bilan des recherches actuelles, l'ACS a choisi de recommander au moins 45 minutes d'AP d'intensité modérée, mais de préférence élevée, au moins cinq jours par semaine, pour réduire le risque de cancer. L'USDHHS recommande aux adultes au moins 30 minutes d'AP d'intensité modérée la plupart des jours de la semaine pour réduire leur risque de maladies chroniques. Cependant, il recommande aussi aux adultes de pratiquer une activité d'intensité modérée à élevée pendant 60 minutes la plupart des jours de la semaine pour mieux prendre en charge leur poids et prévenir la prise de poids, et 60 à 90 minutes d'activité d'intensité modérée à élevée chaque jour pour ne pas reprendre le poids perdu et pour réduire ainsi le risque de maladies chroniques, dont le cancer, associées à l'excès de poids et à l'obésitée ${ }^{2}$. Très récemment, le WCRF/AICR a procédé à un bilan exhaustif des données existantes et recommande maintenant aux adultes au moins 60 minutes d'activité modérée ou 30 minutes ou plus d'activité d'intensité élevée chaque jour pour réduire leur risque de cancer $^{21}$.

Nous nous sommes servis des données du Tomorrow Project ${ }^{22}$, étude de cohorte menée en Alberta, pour estimer le pourcentage d'Albertains qui se conformaient aux directives en matière d'AP pour la prévention du cancer. Vu le très petit nombre d'études menées pour évaluer le niveau d'AP nécessaire pour prévenir le cancer, nous avons aussi exploré les associations possibles entre les caractéristiques personnelles et démographiques et la conformité aux directives en matière d'AP pour la prévention du cancer.

\section{Méthodologie}

\section{Échantillon}

Le Population Research Laboratory de l'Université de l'Alberta a recruté les participants au Tomorrow Project dans toutes les régions géographiques de l'Alberta au moyen de la méthode de composition aléatoire ${ }^{23}$. Cette méthode a été choisie pour l'échantillonnage aléatoire de la population parce que $97 \%$ des ménages de l'Alberta possédaient au moins une ligne téléphonique en $2000^{24}$. Les participants ont été recrutés dans plus de 400 villes et villages et dans toutes les régions rurales de la province afin de constituer un échantillon géographiquement représentatif ${ }^{22}$.

Au total, 29270 Albertains de 35 à 65 ans ont été recrutés dans le Tomorrow Project entre 2001 et 2005, soit $49 \%$ des 59735 personnes admissibles qui ont répondu positivement à l'appel téléphonique; le nombre de personnes admissibles n'ayant pas répondu aux appels téléphoniques est inconnu, de sorte qu'il est impossible de calculer le taux de réponse. Des données complètes sur les facteurs de risque liés au mode de vie étaient disponibles pour 16040 des 29270 personnes recrutées. Au total, 1746 participants ont été exclus de cette étude parce qu'ils répondaient à l'un des critère d'exclusion suivants : transgenre $(\mathrm{n}=2)$, plus de 65 ans $(\mathrm{n}=1328)$, grossesse en cours $(\mathrm{n}=55)$, diagnostic antérieur de cancer $(\mathrm{n}=188)$, non résident de l'Alberta $(\mathrm{n}=75)$ et poids insuffisant ( $\mathrm{n}=98)$. Les données sur les 14294 participants au Tomorrow Project restants ont été utilisées pour l'analyse. Nous pouvons en conclure que le taux de réponse ne peut pas dépasser 25 \% (14 294/[59 735-1 746]).

L'étude a été approuvée par le comité d'éthique de l'Université de Calgary et celui de l'ancien Alberta Cancer Board, maintenant intégré à l'Alberta Health Services-Alberta Cancer Research Ethics Committee.

\section{Collecte des données}

Les Albertains qui ont accepté de participer au Tomorrow Project ont rempli les questionnaires qu'ils ont reçus par la poste au sujet de leurs facteurs de risque liés au mode de vie et de leurs expositions. Pour cette étude, les données recueillies au moyen du Past Year Total Physical Activity Questionnaire (PYTPAQ) [questionnaire sur l'activité physique totale de l'année précédente] et du Health and Lifestyle Questionnaire (HLQ) 
[questionnaire sur la santé et le mode de vie] ont été analysées. Le PYTPAQ est un questionnaire auto-administré valide et fiable qui sert à recueillir des données sur la fréquence, la durée et l'intensité de l'activité physique domestique, de travail, de transport actif et de loisir au cours des douze mois précédents ${ }^{25}$. Le PYTPAQ a été corrélé à des registres d'activités sur sept jours (corrélation de Spearman $[\rho]=0,41$ ) et à des mesures d'accélérométrie de sept jours $(\rho=0,26)$. Le HLQ a été conçu à partir de questionnaires préexistants, notamment ceux utilisés dans l'Enquête sur la santé dans les collectivités canadiennes (ESCC), cycle 1.126, le Prostate, Lung, Colorectal and Ovarian Cancer Screening Trial27 [essai sur le dépistage du cancer de la prostate, du poumon, colorectal et de l'ovaire] et l'Étude prospective européenne sur la nutrition et le cancer $(\text { EPIC })^{28}$ pour évaluer les antécédents médicaux, les antécédents familiaux, les pratiques en matière de dépistage du cancer, le tabagisme, le stress, le soutien social et les caractéristiques personnelles.

Le paramètre pertinent pour cette étude était une activité physique de loisir suffisante pour être conforme aux recommandations en matière d'AP de la SCPE, de l'ACS, de l'USDHHS et du WCRF/AICR. Bien que l'activité de travail, domestique et de transport puisse aussi contribuer à la santé générale, l'activité de loisir est le type d'activité le plus modifiable et il constitue la principale cible des programmes visant à promouvoir la santé publique par l'AP15,19,21. Quatre variables de résultat ont été définies à partir des données recueillies à l'aide du PYTPAQ. Des valeurs en équivalents métaboliques (MET), rapport entre la dépense énergétique pendant une activité et la dépense énergétique du métabolisme au repos ${ }^{18}$, ont été attribuées à chaque activité signalée (de loisir, domestique, de travail et de transport actif) à l'aide du Compendium of Physical Activities ${ }^{29}$. Les valeurs déclarées en ce qui concerne la fréquence et la durée de chaque activité distincte d'une intensité de 3 MET ou plus (considérée comme d'intensité modérée) ont été multipliées pour obtenir une estimation globale des heures hebdomadaires d'AP d'intensité modérée ou élevée. Les variables de résultat ont été définies comme suit :

- satisfaire aux directives de la SCPE d'au moins 2,5 heures par semaine d'activité d'intensité modérée à élevée;

- satisfaire aux directives de l'ACS d'au moins 3,75 heures/semaine d'activité d'intensité modérée à élevée;

- satisfaire aux directives du WCRF/ AICR d'au moins 7 heures/semaine d'activité d'intensité modérée ou 3,5 heures/semaine d'activité d'intensité élevée;

- satisfaire aux directives de l'USDHHS d'au moins 5 heures/semaine d'activité d'intensité modérée à élevée pour prévenir la prise de poids.

Les variables explicatives pertinentes obtenues grâce au HLQ comprenaient l'âge, le sexe, le revenu annuel du ménage, le niveau de scolarité, l'état matrimonial, l'emploi, les maladies chroniques préexistantes (hypertension, hypercholestérolémie et diabète), l'état de santé auto-évalué, le tabagisme, le soutien social (déterminé à l'aide du questionnaire Social Support Survey de la Medical Outcomes Study) ${ }^{30}$ et la résidence en milieu urbain ou rural (d'après le code postal).

\section{Analyse statistique}

Une analyse univariée a permis d'obtenir une description globale de l'échantillon et d'estimer le pourcentage de sujets qui satisfaisaient à chacune des directives en matière d'AP. Nous avons eu recours au test de Cochran-Armitage pour évaluer les tendances dans les proportions qui satisfaisaient à chacune des directives.

Les caractéristiques de l'échantillon d'étude et de la population albertaine, définies à l'aide des données du recensement canadien de $2001^{31}$, ont été comparées pour évaluer la représentativité de l'échantillon. La prévalence du tabagisme et le statut pondéral ont été obtenus au moyen de l'ESCC 2.1. Le taux de réponse à l'ESCC 2.1 était d'environ $83 \%$ en Alberta en 2003 et reflétait les estimations de santé de la population $^{32}$. Un fichier de conversion des codes postaux de Statistique Canada a été utilisé pour coder les participants selon leur région sanitaire. Les poids de l'échantillon ont été estimés au moyen des poids de répartition des groupes d'âge et des sexes selon la région sanitaire de résidence ainsi que selon le niveau de scolarité et le revenu annuel du ménage en fonction des données du recensement canadien de 2001. La proportion de l'échantillon qui se conformait à chacune des directives a ensuite été pondérée de façon à obtenir des estimations du pourcentage d'Albertains qui satisfaisaient à chacune des directives.

Une analyse de régression logistique a été effectuée pour explorer les corrélats possibles de conformité entre chacune des directives. Avant la modélisation, nous avons évalué si les données souffraient d'un problème de multicollinéarité ${ }^{33}$. Le choix des variables s'est fait par élimination arrière hiérarchique ${ }^{34}$, en commençant par toutes les variables explicatives disponibles et tous les modèles ajustés selon l'âge, le sexe et l'indice de masse corporelle (IMC). Une procédure de validation croisée à dix sous-ensembles a été utilisée pour éviter le surajustement ${ }^{35}$. Pour chacune des directives, les données ont été divisées en dix sous-ensembles choisis au hasard et la sélection des variables s'est faite à l'aide de chacun des neuf sous-ensembles de l'essai. Le modèle obtenu a été appliqué à un sous-ensemble de test, et cette procédure a été répétée dix fois, jusqu'à ce que chaque sous-ensemble ait été utilisé comme sous-ensemble de test ${ }^{35}$. Les variables choisies dans au moins trois des dix sous-ensembles à un niveau de signification de $p=0,05$ ont été incluses dans les modèles finaux. La moyenne des coefficients estimés et celle de leurs erreurs-types ont été calculées pour chaque sous-ensemble et ont servi à calculer les rapports de cotes (RC) et les intervalles de confiance (IC) à $95 \%$. Les modèles finaux ont été ajustés à tout l'échantillon et ont fait l'objet de diverses évaluations : qualité de l'ajustement (au moyen du test d'Hosmer-Lemeshow), valeur prédictive (au moyen de la fonction d'efficacité du récepteur [ou courbe ROC]) et pertinence de la fonction lien logit ${ }^{36}$. Toutes les analyses statistiques ont été réalisées au moyen du logiciel STATA version 10 (StataCorp LP) ${ }^{37}$. 


\section{Résultats}

\section{Caractéristiques de l'échantillon d'étude}

L'échantillon était composé majoritairement de femmes $(60 \%)$ et la moyenne d'âge était de 49 ans (tableau 1). Les participants avaient pour la plupart un statut socioéconomique élevé, le tiers ayant fréquenté une université ou poursuivi des études supérieures (33 \%) et ayant un revenu annuel pour leur ménage de 80000 \$ ou plus (37\%). La majorité d'entre eux étaient mariés ou vivaient avec un conjoint de fait (77\%), travaillaient $(77,5 \%)$ et vivaient en milieu urbain (80 \%). La majeure partie des participants jugeaient leur santé très bonne ou excellente (61\%), mais un fort pourcentage d'entre eux avaient un excès de poids ou étaient obèses (25\%) (tableau 1). Comparativement à la population de l'Alberta, les participants comptaient un plus grand pourcentage de femmes, étaient plus âgés, mieux éduqués et mieux nantis (tableau 2). Les participants étaient aussi plus nombreux à avoir un excès de poids ou à être obèses et moins nombreux à fumer que la population de l'Alberta (tableau 2). Globalement, l'échantillon était représentatif des neuf anciennes régions sanitaires de l'Alberta.

\section{Conformité aux directives en matière d'activité physique}

Les sujets qui se conformaient aux directives de la SCPE et de l'ACS (respectivement $63 \%$ et $48 \%$ ) avaient des activités de loisir plutôt que des activités domestiques, de travail ou de transport actif (tableau 3). En revanche, davantage de participants se conformaient aux directives de l'USDHHS et du WCRF/AICR grâce à leur activité de travail. Quel que soit le type d'activité, les participants répondaient dans une plus grande proportion aux directives de la SCPE (93\%) et dans une plus faible proportion à celles de l'USDHHS et du WCRF/AICR (respectivement $78 \%$ et $72 \%$ ) (tableau 3).

\section{Prévalence de la conformité aux directives en matière d'activité physique en Alberta}

Après pondération selon l'âge, le sexe et la région sanitaire de résidence, puis selon le niveau de scolarité et le revenu du ménage, nous avons estimé que $55 \%$ de
TABLEAU 1

Caractéristiques de l'échantillon d'étude, Alberta, 2005

\begin{tabular}{|c|c|c|c|}
\hline Variable & $\begin{array}{c}\text { Échantillon entier } \\
\text { n = } 14294 \\
\%\end{array}$ & $\begin{array}{c}\text { Hommes } \\
\mathbf{n}=5729 \\
\%\end{array}$ & $\begin{array}{c}\text { Femmes } \\
\mathbf{n}=\mathbf{8 5 6 5} \\
\%\end{array}$ \\
\hline Âge moyen (É-T), années (n = 13 970) & $48,7(7,9)$ & $48,6(7,9)$ & $48,7(8,0)$ \\
\hline \multicolumn{4}{|l|}{ IMC, \% (n =13 970) } \\
\hline $18,5-24,9 \mathrm{~kg} / \mathrm{m}^{2}$ & 35,4 & 24,6 & 42,7 \\
\hline $25,0-29,9 \mathrm{~kg} / \mathrm{m}^{2}$ & 39,4 & 49,3 & 32,7 \\
\hline $30,0-39,9 \mathrm{~kg} / \mathrm{m}^{2}$ & 22,5 & 24,7 & 21,0 \\
\hline $40 \mathrm{~kg} / \mathrm{m}^{2}$ ou plus & 2,7 & 1,5 & 3,5 \\
\hline \multicolumn{4}{|l|}{ État matrimonial, \% $(n=14216)$} \\
\hline Marié(e) / conjoint(e) de fait & 76,9 & 81,9 & 75,4 \\
\hline Divorcé(e), séparé(e) ou veuf(ve) & 15,2 & 10,7 & 18,5 \\
\hline Célibataire & 6,5 & 7,3 & 6,1 \\
\hline \multicolumn{4}{|l|}{ Niveau de scolarité, \% (n = 14 005) } \\
\hline Études secondaires & 8,6 & 8,9 & 8,4 \\
\hline Diplôme d'études secondaires & 18,6 & 15,0 & 21,0 \\
\hline Formation technique / collégiale & 39,9 & 40,7 & 39,3 \\
\hline $\begin{array}{l}\text { Études universitaires / diplôme d'études } \\
\text { universitaires }\end{array}$ & 22,9 & 22,7 & 22,9 \\
\hline $3^{\mathrm{e}}$ cycle universitaire & 10,1 & 12,6 & 8,4 \\
\hline \multicolumn{4}{|l|}{ Emploi, \% (n = 14 051) } \\
\hline Emploi à temps plein & 60,7 & 80,6 & 47,4 \\
\hline Emploi à temps partiel & 16,8 & 5,6 & 24,2 \\
\hline Sans emploi & 13,5 & 5,0 & 19,1 \\
\hline Retraité(e) & 8,0 & 7,2 & 8,5 \\
\hline Travailleur(se) autonome & 1,0 & 1,4 & 0,8 \\
\hline \multicolumn{4}{|l|}{ Revenu annuel du ménage, \% ( $n=14$ 022) } \\
\hline Inférieur à $20000 \$$ & 6,0 & 3,6 & 7,5 \\
\hline 20 000-39 999\$ & 16,6 & 12,1 & 19,6 \\
\hline $40000-59999 \$$ & 20,0 & 19,3 & 20,4 \\
\hline $60000-79999 \$$ & 20,1 & 21,6 & 19,1 \\
\hline 80 000-99 999\$ & 14,5 & 16,0 & 13,4 \\
\hline $100000 \$$ ou plus & 22,9 & 27,3 & 19,9 \\
\hline \multicolumn{4}{|l|}{ Lieu de résidence, \% ( $n=14$ 294) } \\
\hline Milieu rural & 19,6 & 19,9 & 20,0 \\
\hline Milieu urbain & 80,4 & 80,1 & 80,0 \\
\hline \multicolumn{4}{|l|}{ État de santé auto-évalué, \% (n = 14 036) } \\
\hline Excellent & 17,3 & 15,8 & 18,3 \\
\hline Très bon & 43,4 & 43,2 & 43,6 \\
\hline Bon & 33,4 & 35,3 & 32,1 \\
\hline Passable & 5,3 & 5,2 & 5,3 \\
\hline Mauvais & 0,6 & 0,4 & 0,7 \\
\hline \multicolumn{4}{|l|}{ Tabagisme, \% ( $\mathrm{n}=14$ 151) } \\
\hline Non-fumeur(se) & 80,5 & 79,8 & 80,9 \\
\hline Fumeur(se) occasionnel(le) & 3,5 & 3,8 & 3,2 \\
\hline Fumeur(se) quotidien(ne) & 16,0 & 16,4 & 15,8 \\
\hline \multicolumn{4}{|l|}{ Hypertension, \% ( $n=14031)$} \\
\hline Oui & 19,5 & 21,7 & 18,0 \\
\hline \multicolumn{4}{|l|}{ Hypercholestérolémie, \% (n = 14 022) } \\
\hline Oui & 24,8 & 29,8 & 21,4 \\
\hline \multicolumn{4}{|l|}{ Diabète, \% ( $\mathrm{n}=14$ 237) } \\
\hline Oui & 3,8 & 4,5 & 3,3 \\
\hline
\end{tabular}

Abréviations : É-T, écart-type; IMC, indice de masse corporelle. 
TABLEAU 2

Comparaison des caractéristiques sociodémographiques de l'échantillon d'étude (2005) et de la population albertaine (données du recensement canadien de 2001)

\begin{tabular}{|c|c|c|}
\hline Caractéristiques sociodémographiques & Échantillon d'étude (\%) & Alberta (\%) \\
\hline \multicolumn{3}{|l|}{ Groupe d'âge, années } \\
\hline $35-39$ & 16,6 & 21,4 \\
\hline $40-44$ & 20,6 & 22,5 \\
\hline $45-49$ & 20,3 & 19,7 \\
\hline $50-54$ & 17,7 & 16,0 \\
\hline $55-59$ & 14,3 & 11,5 \\
\hline $60-64$ & 10,4 & 9,0 \\
\hline \multicolumn{3}{|l|}{ Sexe } \\
\hline Masculin & 40,1 & 50,3 \\
\hline Féminin & 59,9 & 49,7 \\
\hline \multicolumn{3}{|l|}{$I M C^{b}$} \\
\hline $18,5-24,9 \mathrm{~kg} / \mathrm{m}^{2}$ & 35,4 & 43,5 \\
\hline $25,0-29,9 \mathrm{~kg} / \mathrm{m}^{2}$ & 39,4 & 38,1 \\
\hline $30 \mathrm{~kg} / \mathrm{m}^{2}$ ou plus & 25,2 & 18,4 \\
\hline \multicolumn{3}{|l|}{ Tabagisme $^{b}$} \\
\hline Fumeur(se) quotidien(ne) & 16,0 & 24,5 \\
\hline \multicolumn{3}{|l|}{ Niveau de scolarité } \\
\hline Études secondaires & 8,6 & 22,3 \\
\hline Diplôme d'études secondaires & 18,6 & 16,0 \\
\hline Formation technique / collégiale & 39,9 & 29,1 \\
\hline Études universitaires / diplôme d'études universitaires & 22,9 & 27,4 \\
\hline $3^{\mathrm{e}}$ cycle universitaire & 10,1 & 5,1 \\
\hline \multicolumn{3}{|l|}{ Revenu annuel du ménage } \\
\hline$<20000 \$$ & 5,8 & 32,6 \\
\hline $20000-39999 \$$ & 16,2 & 29,3 \\
\hline $40000-59999 \$$ & 19,5 & 19,3 \\
\hline $60000-74999 \$$ & 19,7 & 8,2 \\
\hline$\geq 75000 \$$ & 36,6 & 10,5 \\
\hline
\end{tabular}

${ }^{a}$ Données du recensement canadien de $2001^{31}$.

b Données de l'Enquête sur la santé dans les collectivités canadiennes, Cycle $2.1(2003)^{32}$. la population totale de l'Alberta était assez active pour satisfaire aux directives de la SCPE pour le maintien d'une bonne santé générale. Toutefois, la proportion estimée d'Albertains qui se conformaient aux directives plus rigoureuses de l'ACS, de l'USDHHS et du WCRF/AICR était faible : respectivement $42 \%, 26 \%$ et $23 \%$ (figure 1).

\section{Corrélats de la conformité aux directives en matière d'activité physique grâce à une activité de loisir}

Dans l'ensemble, l'état matrimonial, l'emploi, le revenu annuel du ménage et l'état de santé auto-évalué étaient corrélés à la conformité à toutes les directives en matière d'AP par activité de loisir (tableau 4). Un plus grand pourcentage de personnes divorcées, séparées ou veuves que de personnes mariées ou célibataires se conformaient aux directives de la SCPE $(\mathrm{RC}=1,54$; IC à $95 \%: 1,06$ à 2,26), de l'ACS (RC = 1,63; IC à $95 \%: 1,12$ à 2,35), de l'USDHHS $(\mathrm{RC}=1,62$; IC à $95 \%: 1,08$ à 2,43) et du WCRF/AICR $(\mathrm{RC}=1,51$; IC à $95 \%: 1,09$ à 2,10). Les participants à la retraite respectaient aussi plus fréquemment toutes les directives que ceux qui travaillaient ou étaient sans emploi. La puissance de l'association augmentait avec les exigences accrues des directives : presque trois fois plus de retraités se conformaient aux directives du WCRF/AICR $(\mathrm{RC}=2,76$;

TABLEAU 3

Part de la population à l'étude se conformant aux directives en matière d'activité physique par organisme et par type d'activité physique, Alberta, 2001-2005

\begin{tabular}{|c|c|c|c|c|c|c|c|c|c|}
\hline & \multicolumn{8}{|c|}{ Directives } & \multirow{3}{*}{$\begin{array}{l}\text { Tendance } \\
\text { valeur } \boldsymbol{p}^{\mathrm{e}}\end{array}$} \\
\hline & \multicolumn{2}{|c|}{ SCPE } & \multicolumn{2}{|c|}{$\mathrm{ACS}^{\mathrm{b}}$} & \multicolumn{2}{|c|}{ USDHHS ${ }^{c}$} & \multicolumn{2}{|c|}{ WCRF/AICR ${ }^{\mathrm{d}}$} & \\
\hline & n & $\%$ & $\mathbf{n}$ & $\%$ & $\mathbf{n}$ & $\%$ & $\mathbf{n}$ & $\%$ & \\
\hline \multicolumn{10}{|l|}{ Type d'activitéf } \\
\hline De loisir & 8773 & 62,6 & 6734 & 48,1 & 4115 & 29,5 & 3377 & 24,1 & $<0,0001$ \\
\hline Domestique & 7711 & 55,1 & 6034 & 43,1 & 4156 & 29,8 & 3689 & 26,3 & $<0,0001$ \\
\hline De travail & 5680 & 40,6 & 5387 & 38,5 & 4930 & 35,3 & 4841 & 34,6 & $<0,0001$ \\
\hline
\end{tabular}

Abréviations : ACS, American Cancer Society; AICR, American Institute for Cancer Research; AP, activité physique; SCPE, Société canadienne de physiologie de l'exercice; USDHHS, Department of Health and Human Services des États-Unis; WCRF, World Cancer Research Fund.

${ }^{a}$ Au moins 2,5 heures/semaine d'AP d'intensité modérée à élevée.

${ }^{b}$ Au moins 3,75 heures/semaine d'AP d'intensité modérée à élevée.

c Au moins 5 heures/semaine d'AP d'intensité modérée à élevée pour prévenir la prise de poids.

d Au moins 7 heures/semaine d'AP d'intensité modérée ou 3,5 heures/semaine d'AP d'intensité élevée.

e Évaluation des tendances au moyen du test de Cochran Armitage.

f Activité d'intensité modérée et élevée. 
FIGURE 1

Pourcentage estimé de la population albertaine se conformant aux directives en matière d'activité physique grâce à une activité de loisir, 2001-2005

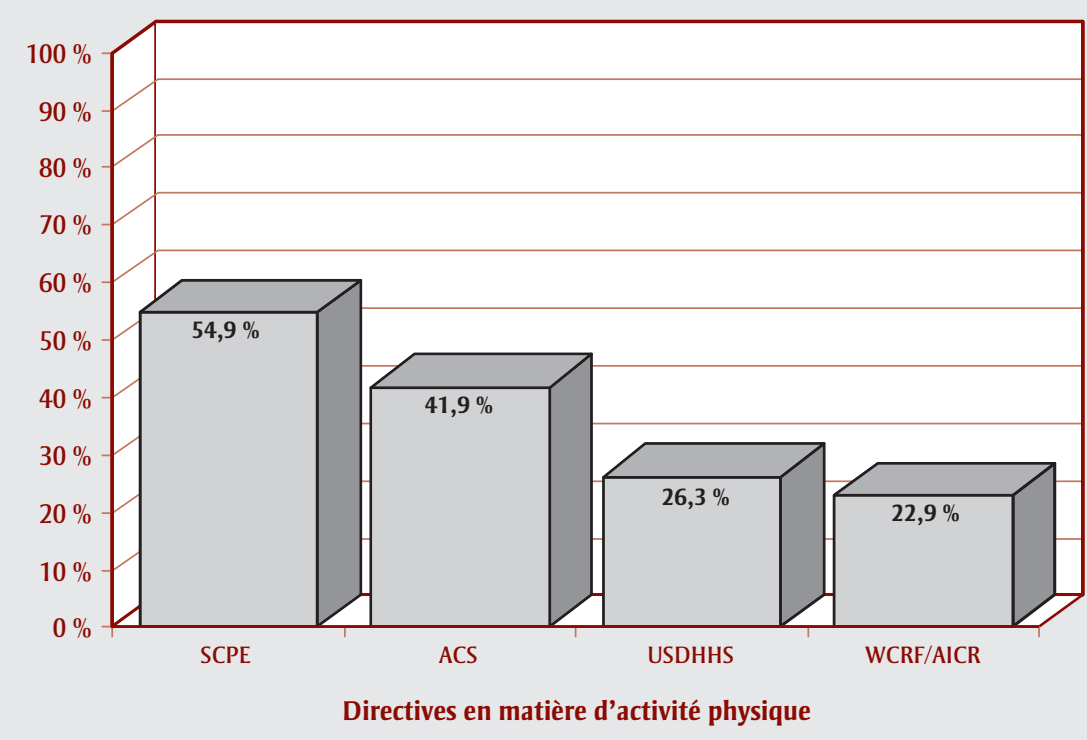

Abréviations : ACS, American Cancer Society; AICR, American Institute for Cancer Research;

SCPE, Société canadienne de physiologie de l'exercice; USDHHS, Department of Health and Human Services des États-Unis; WCRF, World Cancer Research Fund.

Remarque : le pourcentage est pondéré selon la région sanitaire de résidence, l'âge et le sexe, de même que selon le revenu du ménage et le niveau de scolarité (données du recensement de 2001).

IC à $95 \%: 1,57$ à 4,87), comparativement à plus de deux fois plus en ce qui concerne les directives de la SCPE $(\mathrm{RC}=2,30$; IC à $95 \%: 1,32$ à 4,01). En revanche, la puissance de l'association entre un revenu annuel de ménage de 100000 \$ ou plus et la conformité aux directives de la SCPE $(\mathrm{RC}=2,51$; IC à $95 \%: 1,36$ à 4,63$)$ était plus forte qu'elle ne l'était avec les directives du WCRF/AICR ( RC = 1,56; IC à $95 \%: 1,06$ à 3,27$)$. Un nombre significativement plus faible de participants ayant classé leur état de santé comme bon ou inférieur à bon satisfaisaient aux directives de la SCPE $(\mathrm{RC}=0,54$, IC à $95 \%: 0,37$ à 0,80 ), alors qu'un nombre significativement plus faible de participants ayant jugé leur état de santé inférieur à excellent respectaient les directives de l'ACS $(\mathrm{RC}=0,72$; IC à $95 \%: 0,52$ à 0,99$)$, de l'USDHHS (RC $=0,72$; IC à $95 \%: 0,53$ à 0,97 ) et du WCRF/AICR (RC = 0,67; IC à $95 \%$ : 0,47 à 0,95) (tableau 4).

Le sexe et l'IMC étaient les seules caractéristiques significativement associées à une activité suffisante pour satisfaire aux directives pour la prévention du cancer (tableau 4). Moins de femmes que d'hommes se conformaient aux directives de l'ACS $(\mathrm{RC}=0,72$; IC à $95 \%: 0,55$ à 0,93$)$, de l'USDHHS (RC $=0,67$; IC à $95 \%: 0,50$ à 0,89$)$ ou du WCRF/AICR $(\mathrm{RC}=0,63$; IC à $95 \%: 0,47$ à 0,85), mais la conformité aux directives de la SCPE pour le maintien d'une bonne santé générale ne différait pas selon le sexe. L'excès de poids n'était négativement associé qu'avec la conformité aux directives de l'USDHHS $(\mathrm{RC}=0,52$; IC à $95 \%: 0,39$ à 0,70$)$, alors que l'obésité était associée à la conformité à la fois aux directives de l'USDHHS $(\mathrm{RC}=0,45$; IC à $95 \%: 0,32$ à 0,65$)$ et à celles du WCRF/IARC $(\mathrm{RC}=0,79$; IC à $95 \%: 0,63$ à 0,98$)$.

\section{Analyse}

Nos résultats laissent penser que peu d'Albertains font assez d'activité physique de loisir pour réduire leur risque de cancer, probablement du fait des plus hauts niveaux d'activité nécessaires pour se conformer aux directives de l'ACS ou du WCRF/AICR comparativement aux directives de la SCPE pour le maintien d'une bonne santé générale. Les directives du WCRF/AICR exigeant presque trois fois plus de temps que celles de la SCPE, les participants doivent consacrer plus de temps à l'activité physique pour réduire leur risque de cancer. Les personnes retraitées, qui disposent de plus de temps de loisir, étaient de fait plus nombreuses à s'adonner à une activité physique au niveau recommandé pour la prévention du cancer.

Ces résultats sont conformes au modèle des croyances relatives à la santé (Health Belief Model), selon lequel lorsque les obstacles perçus à un comportement s'accroissent, la probabilité d'adoption de ce comportement diminue $^{38}$. Il est probable qu'un niveau d'activité physique de loisir suffisant pour réduire le risque de cancer se heurte à de plus grands obstacles temporels ou à un manque de disponibilité à cause d'autres engagements et exige une plus grande motivation que le niveau d'activité moindre nécessaire pour obtenir des bienfaits pour la santé générale. De plus, les directives de la SCPE sont régulièrement communiquées aux Canadiens depuis $1998^{15}$. Les Canadiens qui souhaitent être actifs s'efforcent sans doute de se conformer à ces directives pour le maintien d'une bonne santé générale sans savoir qu'une plus grande activité est nécessaire pour réduire le risque de cancer. Les avantages perçus, autre composante du modèle des croyances relatives à la santé, sont également importants pour encourager un comportement ${ }^{38}$ : si les directives en matière d'AP pour prévenir le cancer étaient mieux connues, les individus seraient sans doute encouragés à s'en servir comme référence en matière d'AP.

Dans notre étude, moins de femmes que d'hommes pratiquaient des activités physiques de loisir aux niveaux recommandés pour réduire le risque de cancer, et ce résultat était comparable aux résultats d'autres rapports ${ }^{39-41}$, même une fois pris en compte d'autres facteurs sociodémographiques. Un certain nombre de facteurs contextuels culturels et sociaux, comme les rôles sexuels, entraînent des différences de comportement en matière d'AP entre les hommes et les femmes ${ }^{40-44}$. Les facteurs de motivation sont également différents : les femmes indiquent plus souvent que leur image corporelle, leur apparence et les inquiétudes concernant leur santé sont 
TABLEAU 4

Rapports de cotes estimés de la conformité aux directives en matière d'activité physique grâce à une activité de loisir, Alberta, 2001-2005

\begin{tabular}{|c|c|c|c|c|c|c|c|c|}
\hline \multirow{2}{*}{$\begin{array}{l}\text { Variable } \\
\text { Âge, années }\end{array}$} & \multicolumn{8}{|c|}{ Directives } \\
\hline & \multicolumn{2}{|c|}{$\begin{array}{c}\text { SCPE }^{b} \\
\text { RC }^{\text {a }}(\text { IC à } 95 \%)\end{array}$} & \multicolumn{2}{|c|}{$\begin{array}{c}\text { ACS }^{c} \\
\left.\text { RCa }^{(I C} \text { à } 95 \%\right)\end{array}$} & \multicolumn{2}{|c|}{$\begin{array}{c}\text { USDHHS }^{\mathrm{d}} \\
\left.\text { RCa }^{\mathrm{a}} \text { (IC à } 95 \%\right)\end{array}$} & \multicolumn{2}{|c|}{$\begin{array}{c}\text { WCRF/AICR } \\
\text { RC }^{\mathbf{a}}(\text { IC à } 95 \%)\end{array}$} \\
\hline $35-39$ & 1,00 & & 1,00 & & 1,00 & & 1,00 & \\
\hline $40-44$ & 1,02 & $(0,69$ à 1,51$)$ & 0,99 & $(0,68$ à 1,44$)$ & 0,94 & $(0,63$ à 1,41) & 0,91 & (0,60 à 1,39) \\
\hline $45-49$ & 0,98 & $(0,66$ à 1,44$)$ & 0,98 & $(0,68$ à 1,43$)$ & 0,91 & $(0,61$ à 1,37$)$ & 0,87 & $(0,57$ à 1,34$)$ \\
\hline $50-54$ & 0,81 & $(0,54$ à 1,22$)$ & 0,86 & $(0,58$ à 1,27) & 0,86 & $(0,56$ à 1,32) & 0,82 & (0,53 à 1,29) \\
\hline $55-59$ & 0,80 & $(0,52$ à 1,25$)$ & 0,80 & $(0,52$ à 1,24$)$ & 0,74 & $(0,46$ à 1,21$)$ & 0,75 & $(0,45$ à 1,24$)$ \\
\hline $60-65$ & 0,75 & $(0,44$ à 1,28$)$ & 0,72 & $(0,42$ à 1,22) & 0,72 & $(0,40$ à 1,29) & 0,67 & $(0,36$ à 1,24) \\
\hline \multicolumn{9}{|l|}{ Sexe } \\
\hline Masculin & 1,00 & & 1,00 & & 1,00 & & 1,00 & \\
\hline Féminin & 0,85 & $(0,65$ à 1,11$)$ & 0,72 & $(0,55$ à 0,93$)$ & 0,67 & $(0,50$ à 0,89$)$ & 0,63 & $(0,47$ à 0,85$)$ \\
\hline \multicolumn{9}{|l|}{$\mathrm{IMC}, \mathrm{kg} / \mathrm{m}^{2}$} \\
\hline $18,5-24,9$ & 1,00 & & 1,00 & & 1,00 & & 1,00 & \\
\hline $25,0-29,9$ & 0,96 & $(0,72$ à 1,28$)$ & 0,94 & (0,71 à 1,23) & 0,52 & $(0,39$ à 0,70$)$ & 0,96 & $(0,71$ à 1,31$)$ \\
\hline 30,0 ou plus & 0,82 & $(0,60$ à 1,14$)$ & 0,83 & $(0,60$ à 1,13$)$ & 0,45 & $(0,32$ à 0,65$)$ & 0,79 & $(0,63$ à 0,98$)$ \\
\hline \multicolumn{9}{|l|}{ État matrimonial } \\
\hline Marié(e) / conjoint(e) de fait & 1,00 & & 1,00 & & 1,00 & & 1,00 & \\
\hline Divorcé(e), séparé(e) ou veuf(ve) & 1,54 & $(1,06$ à 2,26$)$ & 1,63 & $(1,12$ à 2,35$)$ & 1,62 & (1,08 à 2,43) & 1,51 & $(1,09$ à 2,10$)$ \\
\hline Célibataire & 1,41 & $(0,84$ à 2,36$)$ & 1,50 & $(0,90$ à 2,49$)$ & 1,52 & $(0,87$ à 2,66$)$ & 1,52 & $(0,85$ à 2,71$)$ \\
\hline \multicolumn{9}{|l|}{ Niveau de scolarité } \\
\hline Études secondaires & 1,00 & & 1,00 & & 1,00 & & 1,00 & \\
\hline Diplôme d'études secondaires & 1,20 & (0,73 à1,95) & 1,06 & (0,64 à1,74) & 0,91 & $(0,52$ à 1,61$)$ & 1,00 & $(0,55$ à 1,81$)$ \\
\hline Formation technique / collégiale & 1,28 & $(0,81$ à 2,02$)$ & 1,10 & (0,69 à1,75) & 0,90 & $(0,53$ à 1,53$)$ & 0,93 & $(0,54$ à 1,61$)$ \\
\hline $\begin{array}{l}\text { Études universitaires / diplôme d'études } \\
\text { universitaires }\end{array}$ & 1,40 & $(0,85$ à 2,30$)$ & 1,22 & $(0,74$ à 2,01$)$ & 1,03 & $(0,59$ à 1,81$)$ & 1,12 & $(0,62$ à 2,01$)$ \\
\hline $3^{\mathrm{e}}$ cycle universitaire & 1,40 & $(0,78$ à 2,53$)$ & 1,08 & (0,61 à1,92) & 0,91 & $(0,48$ à 1,71$)$ & 0,97 & $(0,50$ à 1,87$)$ \\
\hline \multicolumn{9}{|l|}{ Emploi } \\
\hline Emploi à temps plein & 1,00 & & 1,00 & & 1,00 & & 1,00 & \\
\hline Emploi à temps partiel & 1,19 & $(0,84$ à 1,69$)$ & 1,27 & (0,90 à 1,78) & 1,19 & (0,81 à 1,73) & 1,11 & (0,74 à 1,67) \\
\hline Sans emploi & 1,19 & (0,81 à 1,75) & 1,27 & (0,87 à 1,86) & 1,40 & $(0,92$ à 2,13) & 1,41 & (0,91 à 2,18) \\
\hline Retraité(e) & 2,30 & $(1,32$ à 4,01$)$ & 2,65 & $(1,56$ à 4,48) & 3,04 & $(1,74$ à 5,31$)$ & 2,76 & (1,57 à 4,87) \\
\hline Travailleur(se) autonome & 0,74 & $(0,21$ à 2,62$)$ & 0,76 & $(0,21$ à 2,77$)$ & 0,97 & $(0,22$ à 4,19$)$ & 1,20 & (0,29 à 4,99) \\
\hline \multicolumn{9}{|l|}{ Revenu annuel du ménage } \\
\hline Inférieur à $20000 \$$ & 1,00 & & 1,00 & & 1,00 & & 1,00 & \\
\hline $20000-39999 \$$ & 1,16 & $(0,66$ à 2,04$)$ & 1,14 & $(0,64$ à 2,04$)$ & 1,06 & $(0,55$ à 2,06$)$ & 0,99 & (0,49 à 1,98) \\
\hline $40000-59999 \$$ & 1,26 & $(0,71$ à 2,22$)$ & 1,27 & $(0,71$ à 2,26$)$ & 1,16 & (0,60 à 2,25) & 1,03 & $(0,51$ à 2,06$)$ \\
\hline $60000-79999 \$$ & 1,62 & (0,90 à 2,90) & 1,52 & (0,84 à 2,75) & 1,39 & (0,71 à 2,74) & 1,20 & (0,59 à 2,43) \\
\hline 80 000-99 $999 \$$ & 1,80 & (0,97 à 3,34) & 1,79 & $(1,17$ à 2,75$)$ & 1,58 & $(0,78$ à 3,20) & 1,30 & $(0,62$ à 2,73$)$ \\
\hline $100000 \$$ ou plus & 2,51 & $(1,36$ à 4,63) & 2,43 & $(1,32$ à 4,48$)$ & 2,05 & $(1,03$ à 4,08$)$ & 1,56 & $(1,06$ à 3,27$)$ \\
\hline \multicolumn{9}{|l|}{ État de santé auto-évalué } \\
\hline Excellent & 1,00 & & 1,00 & & 1,00 & & 1,00 & \\
\hline Très bon & 0,75 & $(0,52$ à 1,08$)$ & 0,72 & $(0,52$ à 0,99$)$ & 0,72 & (0,53 à 0,97) & 0,67 & $(0,47$ à 0,95$)$ \\
\hline Bon & 0,54 & $(0,37$ à 0,80$)$ & 0,52 & $(0,36$ à 0,74$)$ & 0,50 & $(0,34$ à 0,73$)$ & 0,47 & $(0,32$ à 0,71$)$ \\
\hline Passable ou mauvais & 0,38 & $(0,21$ à 0,71$)$ & 0,36 & $(0,19$ à 0,67$)$ & 0,37 & $(0,17$ à 0,78$)$ & 0,40 & $(0,19$ à 0,85$)$ \\
\hline \multicolumn{9}{|l|}{ Tabagisme } \\
\hline Non-fumeur(se) & 1,00 & & 1,00 & & 1,00 & & 1,00 & \\
\hline Fumeur(se) occasionnel(le) & 1,14 & $(0,58$ à 2,23) & 1,17 & $(0,62$ à 2,22) & 1,16 & $(0,58$ à 2,31$)$ & 1,13 & (0,56 à 2,32) \\
\hline Fumeur(se) quotidien(ne) & 0,65 & $(0,47$ à 0,90$)$ & 0,70 & (0,50 à 0,97) & 0,77 & $(0,53$ à 1,13$)$ & 0,82 & $(0,55$ à 1,22$)$ \\
\hline Soutien socialf $f$ & 1,12 & $(0,96$ à 1,31$)$ & 1,09 & $(0,94$ à 1,28$)$ & 1,08 & $(0,91$ à 1,28$)$ & 1,04 & $(0,87$ à 1,25$)$ \\
\hline
\end{tabular}

Abréviations : ACS, American Cancer Society; AICR, American Institute for Cancer Research; AP, activité physique; IC, intervalle de confiance; RC, rapport de cotes;

SCPE, Société canadienne de physiologie de l'exercice; USDHHS, Department of Health and Human Services des États-Unis; WCRF, World Cancer Research Fund.

${ }^{a}$ Estimé par régression logistique au moyen d'une méthode de validation croisée à dix sous-ensembles.

${ }^{b}$ Au moins 2,5 heures/semaine d'AP d'intensité modérée à élevée.

c Au moins 3,75 heures/semaine d'AP d'intensité modérée à élevée.

d Au moins 5 heures/semaine d'AP d'intensité modérée à élevée pour prévenir la prise de poids.

e Au moins 7 heures/semaine d'AP d'intensité modérée ou 3,5 heures/semaine d'AP d'intensité élevée.

${ }^{\mathrm{f}}$ Déterminé à l'aide du questionnaire Social Support Survey de la Medical Outcomes Study ${ }^{30}$. 
des raisons d'être physiquement actives d'importance égale ${ }^{45-48}$. Ces résultats donnent à penser que les différences liées au sexe doivent être prises en considération lorsqu'on veut inciter la population à faire plus d'activité physique pour prévenir le cancer.

Malgré les différences liées au sexe, tant les hommes que les femmes ayant un excès de poids ou obèses étaient significativement moins nombreux que les personnes ayant un poids normal à se conformer aux directives de l'USDHHS et du WCRF/AICR. Comme ces directives exigent 30 à 60 minutes d'AP de loisir chaque jour, il est possible que les personnes ayant un excès de poids ou obèses soient physiquement incapables de s'adonner à une activité physique d'une durée suffisante ou d'apporter les changements requis à leur mode de vie pour avoir un tel niveau d'activité. En effet, la probabilité de se soumettre un programme d'AP est plus faible parmi les personnes ayant un excès de poids ou obèses que parmi celles ayant un poids normal, même si ce programme ne comporte que de la marche ${ }^{49-50}$. L'AP peut être particulièrement difficile pour les personnes ayant un excès de poids ou obèses qui ont des douleurs ou une gêne exacerbées par leur poids excessif ${ }^{51}$. En revanche, ces résultats pourraient témoigner $\mathrm{du}$ fait qu'un niveau d'AP suffisant pour satisfaire aux directives de l'USDHHS et du WCRF/AICR aide à perdre du poids et protège contre les effets néfastes d'un poids excessif ${ }^{52-55}$. Nos résultats sont de toute manière comparables à d'autres résultats selon lesquels l'excès de poids et l'obésité sont associés séparément à un faible niveau d'AP ${ }^{51,56-57}$.

Les personnes dans la catégorie du plus haut revenu étaient les plus nombreuses à avoir une activité physique suffisante pour répondre à toutes les directives, constatation qui est également conforme à des études antérieures ${ }^{42,58-59}$. Un faible statut socio-économique est souvent associé à des responsabilités de soignant, du temps consacré aux enfants, un travail physiquement exigeant, l'absence de moyens de transport, un voisinage non sécuritaire, des horaires de travail non flexibles et des déménagements fréquents ${ }^{41}$, facteurs qui peuvent tous empêcher la participation à des activités de loisir. Fait intéressant, la puissance de l'association entre le revenu annuel du ménage et la conformité aux directives en matière d'AP diminuait avec l'augmentation du niveau d'activité nécessaire pour satisfaire aux directives. Cette relation était la plus faible pour la conformité aux directives de l'USDHHS et du WCRF/AICR, ce qui laisse croire que des facteurs personnels plus complexes pourraient entraver l'AP de loisir à un haut niveau. L'affaiblissement de l'association entre l'activité et le revenu pourrait aussi découler du fait que les retraités étaient plus fréquemment membres de la classe moyenne et, par conséquent, plus susceptibles de suivre les directives pour la réduction du risque de cancer. Malgré cette relation plus faible, le revenu était tout de même fortement corrélé à la conformité aux directives pour la prévention du cancer.

Cette étude est l'une des premières à évaluer la prévalence de l'AP à un niveau suffisant pour prévenir le cancer. Jusqu'à maintenant, les estimations de l'AP chez les Canadiens étaient fondées sur les directives de la SCPE relatives à une activité suffisante pour être bénéfique pour la santé. En utilisant la même approche, l'ESCC (cycle 2.1) a produit des estimations selon lesquelles, durant la période de l'étude, $48 \%$ des Canadiens ${ }^{60}$ et $52 \%$ des Albertains $^{61}$ de 35 à 65 ans étaient physiquement actifs ${ }^{61}$. Nous avons estimé dans notre étude que $63 \%$ des Albertains étaient assez actifs pour satisfaire aux directives de la SCPE (tableau 3). Cette différence dans les estimations a persisté même après l'ajustement selon l'âge, le sexe, le revenu et le niveau de scolarité, ce qui laisse croire que l'échantillon d'étude diffère de la population de l'Alberta par d'autres facteurs dont il faut tenir compte lorsqu'on estime la prévalence de l'AP dans la population, ce qui est une tâche complexe. Les estimations plus élevées produites par notre étude pourraient aussi être attribuées à l'effet du "participant en bonne santé ». Environ 60 \% des sujets de l'étude jugeaient leur santé très bonne ou excellente, et la prévalence du diabète était plus faible dans l'échantillon que dans la population albertaine $(3,8 \%$, contre $4,9 \%$ chez les Albertains ${ }^{61}$ ) tout comme celle du tabagisme (16\% des sujets de l'étude fumaient chaque jour, contre $25 \%$ des
Albertains). Même si l'excès de poids et l'obésité étaient plus répandus parmi les participants à l'étude, ces derniers semblaient en meilleure santé que l'ensemble des Albertains et semblaient plus nombreux à pratiquer une AP de loisir. Les différences dans les mesures de l'AP de loisir entre l'ESCC et le PYTPAQ employées dans notre étude pourraient aussi expliquer les différences dans l'estimation de la prévalence. L'ESCC utilise un élément de questionnaire en plusieurs parties pour mesurer la fréquence et la durée de la participation à une liste donnée d'activités de loisir au cours des trois derniers mois ${ }^{62}$. Le PYTPAQ, lui, évalue les activités de loisir au cours de la dernière année au moyen d'une approche plus détaillée, qui permet aux participants d'indiquer la durée, la fréquence et l'intensité de toutes leurs activités récréatives et sportives. Le PYTPAQ reflète probablement mieux les profils d'activité habituels, alors que, en raison de la période plus courte sur laquelle porte le questionnaire de l'ESCC, les réponses peuvent être davantage influencées par les variations saisonnières ou une maladie aiguë ${ }^{63}$.

De vastes enquêtes en population générale ont permis d'estimer que durant la période d'étude 45,9 \% des adultes des États-Unis ${ }^{64}$ et $29 \%$ des adultes de 15 pays de l'Union européenne $^{65}$ pratiquaient des activités physiques d'intensité modérée à élevée pendant 150 minutes chaque semaine. De même, dans le cadre d'une étude récente, les chercheurs ont estimé que $15 \%$ des Canadiens adultes avaient ce niveau d'activité ${ }^{66}$. Cependant, comme ces estimations englobaient l'AP de travail, de transport et domestique en plus de l'AP de loisir, il est difficile de les comparer à nos propres estimations, qui ne portent que sur l'AP de loisir. L'AP totale a été utilisée pour estimer la prévalence de l'AP dans différentes régions ${ }^{64,67-69}$, mais l'accent mis sur l'AP de loisir est valable, car ce type d'activité est le plus susceptible d'être modifié, contrairement à l'activité de travail ou domestique. Certaines données montrent que le risque de cancer du sein, de la prostate et de l'endomètre et du cancer colorectal est significativement abaissé lorsqu'on entreprend une AP de plus forte intensité ${ }^{6,8,70}$. Cela s'explique probablement par une modification des biomarqueurs de 
l'inflammation, de la résistance à l'insuline et des taux d'hormones sexuelles et d'hormones métaboliques, qui favorisent une réduction du risque de cancer en réponse aux activités de loisir d'intensité modérée ou élevée mais pas en réponse aux activités domestiques de faible intensité ${ }^{12,71}$. L’AP de loisir est donc une cible logique des interventions sanitaires dans la population visant à prévenir le cancer.

\section{Limites de l'étude}

Notre étude comportait certaines limites, dont l'interprétation des résultats. Même s'il s'agit d'une caractéristique courante des études par composition aléatoire, le taux de réponse était bas, moins de $25 \%$, et l'échantillon non pondéré n'était pas représentatif de la population albertaine. Même si nous avons essayé de pondérer les estimations de la prévalence de façon à ce qu'elles correspondent davantage à celles de la population de l'Alberta, la généralisation de nos résultats peut être limitée. Les données relatives à l'AP étaient auto-déclarées, ce qui a pu entraîner une surdéclaration des niveaux d'activité en raison d'un biais de désirabilité sociale. Des erreurs de mesure et des estimations inexactes ont aussi pu se produire parce qu'il peut être difficile de se souvenir d'une $\mathrm{AP}^{18}$ : les participants à notre étude devaient se rappeler leur pratique d'AP sur une période d'un an. Toutefois, le PYTPAQ s'est révélé un outil valide et fiable dans un vaste échantillon d'hommes et de femmes choisis au hasard ${ }^{25}$. Notre utilisation d'instruments déjà validés et d'une fiabilité éprouvée pour mesurer l'AP et toutes les autres variables a aidé à réduire au minimum les erreurs de mesure ${ }^{25}$. Par ailleurs, le caractère transversal de notre étude limite l'interprétation des résultats aux corrélations et ne permet pas d'associations causales. Néanmoins, ces résultats ont permis d'identifier des facteurs qui méritent une étude plus poussée à titre de cibles importantes pour les interventions visant à augmenter l'AP dans une optique de prévention du cancer dans la population.

\section{Recommandations}

Étant donné que $42 \%$ des Albertains ne s'adonnent pas à une activité physique suffisante pour avoir des effets bénéfiques sur leur santé générale, les interventions à venir devraient encourager les personnes sédentaires à faire de l'exercice. Ces interventions devraient promouvoir un niveau plus élevé d'activité pour obtenir des bienfaits additionnels, soit la prévention du cancer, dans ce segment de la population tout comme parmi les personnes déjà actives. En 2005, les Canadiens consacraient environ 6 heures chaque jour à des activités de loisir, que ce soit regarder la télévision, surfer sur Internet ou s'adonner à des hobbies, que ces derniers impliquent ou non une activité physique ${ }^{72}$. Cette panoplie d'activités de loisir rend difficile la promotion de l'activité physique. Elle met aussi en lumière la nécessité de mettre en œuvre des interventions efficaces pour renforcer les facteurs favorisant l'activité physique et réduire les obstacles s'y opposant.

Les directives nationales actuelles pourraient être insuffisantes tant pour la prévention du cancer que pour la prise en charge du poids ${ }^{21}$. Vu l'abondance des données indiquant que l'obésité contribue au risque de cancer, l'incitation à faire assez d'activité physique pour perdre du poids et gérer ce dernier pourrait être une cible importante des stratégies de prévention du cancer dans la population. Par ailleurs, la quantité nécessaire d'AP est mal définie, d'où les écarts entre les directives. Lélaboration des directives est tributaire des recherches établissant un lien entre l'AP et le cancer ${ }^{19-21}$, recherches qui consistent principalement en études d'observation de types variés ${ }^{73}$. Des essais randomisés sont nécessaires pour formuler des recommandations définitives relativement à la quantité d'exercice requise et, tant que de tels essais ne sont pas réalisés, il pourrait s'avérer plus prudent de fournir un ensemble progressif de directives qui soulignent les bienfaits pour la santé associés à chaque niveau et intensité d'AP, particulièrement les niveaux entraînant une plus forte réduction du risque de cancer $^{74}$. Enfin, d'autres recherches sont nécessaires pour mettre en œuvre des interventions efficaces de promotion de l'AP qui tiennent compte des facteurs de motivation individuels ainsi que des facteurs sociaux et environnementaux favorisant l'AP.

\section{Remerciements}

Ce projet a été financé par l'Alberta Cancer Foundation. Fabiola Aparicio-Ting bénéficiait d'une bourse de doctorat de la Société canadienne du cancer. Christine Friedenreich bénéficiait de fonds associés au prix Health Senior Scholar de l'Alberta Heritage Foundation for Medical Research. Ronald Plotnikoff était subventionné par l'entremise d'une chaire de recherche appliquée en santé publique des Instituts de recherche en santé du Canada. Les auteurs souhaitent remercier chaleureusement Paula Robson, chercheure principale du Tomorrow Project, pour leur avoir communiqué les données de base sur cette cohorte. Ils souhaitent aussi exprimer leurs remerciements à Heather Whelan et à Will Rosner pour les avoir aidés à accéder aux ensembles de données du Tomorrow Project et à les utiliser.

\section{Références}

1. Comité directeur de la Société canadienne du cancer. Statistiques canadiennes sur le cancer 2011 : section spéciale sur le cancer colorectal. Toronto (Ont.) : Société canadienne du cancer; 2011.

2. Institut national du cancer du Canada. Statistiques canadiennes sur le cancer 2004. Toronto (Ont.) : Institut national du cancer du Canada; 2004.

3. Colditz GA, Sellers TA, Trapido E. Epidemiology - identifying the causes and preventability of cancer? Nat Rev Cancer. 2006;6(1):75-83.

4. Stein CJ, Colditz GA. Modifiable risk factors for cancer. Brit J Cancer. 2004;90(2):299-303.

5. Courneya KS, Friedenreich CM, editors. Physical activity and cancer. Berlin (DE): Springer; 2011.

6. Wolin KY, Yan Y, Colditz GA, Lee IM. Physical activity and colon cancer prevention: a meta-analysis. Br J Cancer. 2009;100(4):611-6.

7. Cust AE. Physical activity and gynecologic cancer prevention. Dans : Courneya KS, Friedenreich CM, editors. Physical activity and cancer. New York (NY): Springer; 2011. p. 159-88. 
8. Monninkhof EM, Elias SG, Vlems FA, van der Tweel I, Schuit AJ, Voskuil DW et collab. Physical activity and breast cancer: a systematic review. Epidemiology. 2007;18(1):137-57.

9. Leitzmann MF. Physical activity and genitourinary cancer prevention. Dans : Courneya KS, Friedenreich CM (dir.). Physical activity and cancer. New York (NY): Springer; 2011. p. 43-72.

10. Rogers CJ, Colbert LH, Greiner JW, Perkins SN, Hursting SD. Physical activity and cancer prevention: pathways and targets for intervention. Sports Med. 2008;38(4):271-96.

11. Neilson HK, Friedenreich CM, Brockton NT, Millikan RC. Physical activity and postmenopausal breast cancer: proposed biologic mechanisms and areas for future research. Cancer Epidemiol Biomarkers Prev. 2009;18(1):11-27.

12. McTiernan A. Mechanisms linking physical activity with cancer. Nat Rev Cancer. 2008;8(3):205-11.

13. Renehan AG, Soerjomataram I, Leitzmann MF. Interpreting the epidemiological evidence linking obesity and cancer: a framework for populationattributable risk estimations in Europe. Eur J Cancer. 2010;46(14):2581-92.

14. Wolin KY, Carson K, Colditz GA. Obesity and cancer. Oncologist. 2010;15(6):556-65.

15. Sharratt MT, Hearst WE. Canada's physical activity guides: background, process, and development. Can J Public Health. 2007;98(2):S9-15.

16. Société canadienne de physiologie de l'exercice. Directives canadiennes en matière d'activité physique à l'intention des adultes âgés de 18 à 64 ans [Internet]. Ottawa (Ont.) : SCPE; 2003 [consulté le 14 oct. 2003]. PDF (61 Ko) téléchargeable à partir du lien : http://www.csep.ca/CMFiles /Directives/CSEP-InfoSheets-adults-FR.pdf

17. Sallis JF, Owen N. Physical activity and behavioral medicine. Thousand Oaks, (CA): SAGE Publications, Inc.; 1999. Chapitre 5, Measuring physical activity; p. 71-91.
18. Pettee KK, Storti KL, Ainsworth BE, Kriska AM. Measurement of physical activity and inactivity in epidemiological studies. Dans : Lee IM (dir.). Epidemiologic methods in physical activity studies. New York, NY: Oxford University Press; 2009. p. 15-33.

19. Byers T, Nestle M, McTiernan A, Doyle C, Currie-Williams A, Gansler $\mathrm{T}$ et collab. American Cancer Society guidelines on nutrition and physical activity for cancer prevention: reducing the risk of cancer with healthy food choices and physical activity. CA Cancer J Clin. 2002;52(2):92-119.

20. U.S. Department of Health and Human Services and U.S. Department of Agriculture. Dietary guidelines for Americans, 2005. Washington (DC): U.S. Department of Health and Human Services; 2005. Joint publication of the U.S. Department of Agriculture; 2005.

21. World Cancer Research Fund and the American Institute for Cancer Research. Food, nutrition, physical activity and the prevention of cancer: a global perspective. Washington (DC): American Institute for Cancer Research; 2007.

22. Bryant H, Robson PJ, Ullman R, Friedenreich C, Dawe U. Constitution d'une cohorte en population en Alberta, Canada : une étude de faisabilité. Maladies chroniques au Canada. 2006;27(2):55-65.

23. Lavrakas PJ. Telephone survey methods sampling, selection, and supervision. $2^{\mathrm{e} e ́ d .}$ Thousand Oaks, (CA): SAGE Publications; 1993. Chapitre 2, Random Digit Dialing; p. 33-48.

24. Statistique Canada. Enquête sur le service téléphonique résidentiel (ESTR). Ottawa (Ont.) : Statistique Canada; 2001.

25. Friedenreich CM, Courneya KS, Neilson HK, Matthews CE, Willis G, Irwin M et collab. Reliability and validity of the Past Year Total Physical Activity Questionnaire. Am J Epidemiol. 2006;163:959-70.

26. Statistique Canada. Enquête sur la santé dans les collectivités canadiennes (ESCC) : Cycle 1.1 : enrichissement de données sur la santé au Canada. Consultable en ligne à la page : http://www.statcan.gc.ca/concepts /health-sante/cchs-escc-info-fra.htm
27. National Cancer Institute. A 16-year randomized screening study for prostate, lung, colorectal and ovarian cancer - PLCO Trial [Internet]. Bethesda (MD): National Cancer Institute; 1993 [consulté le 4 mars 2010]. Consultable en ligne à la page : http:// www.cancer.gov/clinicaltrials/PLCO-1

28. International Agency for Research on Cancer. EPIC Project [Internet]. Lyon (FR): World Health Organization; 2010 [consulté le 4 mars 2010]. Consultable en ligne à la page : http://epic.iarc.fr/about.php

29. Ainsworth BE, Haskell WL, Whitt MC, Irwin ML, Swartz AM, Strath SJ et collab. Compendium of physical activities: an update of activity codes and MET intensities. Med Sci Sports Exerc. 2000;32(9 Suppl):S498-504.

30. Sherbourne CD, Stewart AL. The MOS social support survey. Soc Sci Med. 1991;32(6):705-14.

31. Statistique Canada. Recensement du Canada de 2001. Ottawa (Ont.): Statistique Canada; 2003 [consulté le 4 mars 2010]. Consultable en ligne à la page : http://www12.statcan.ca /francais/census01/home/index.cfm

32. Statistique Canada. Enquête sur la santé dans les collectivités canadiennes 2003 : Guide de l'utilisateur du fichier de microdonnées à grande diffusion. Ottawa (Ont.) : Statistique Canada; 2005 [consulté le 4 mars 2010]. PDF (700 Ko) téléchargeable à partir du lien : http:// www23.statcan.gc.ca:81/imdb-bmdi/pub /document/3226_D7_T9_V2-fra.pdf

33. Norman GR, Streiner DL. Biostatistics: the bare essentials. $2^{\mathrm{e}}$ éd. Hamilton, (Ont): BC Decker; 2000. Chapitre 14, Multiple regression; p. 127-38.

34. Kleinbaum DG, Klein M. Logistic regression: a self-learning text. $2^{\mathrm{e}}$ éd. New York, (NY): Springer; 2002. Chapitre 6, Modeling strategy guidelines; p. 161-90.

35. Harrell FE. Regression modeling strategies. New York, (NY): Springer; 2001. Chapitre 5, Resampling, validating, describing, and simplifying the model; p. 87-104.

36. Harrell FE. Regression modeling strategies. New York, (NY): Springer; 2001. Chapitre 10, Binary logistic regression; p. 215-68.

37. StataCorp LP. STATA Release 10.0. College Station (TX): Stata Corporation; 2001. 
38. Janz NK, Champion VL, Strecher VJ. The health belief model. In: Glanz K, Rimer BK, Lewis FM, editors. Health behavior and health education: Theory, research and practice.

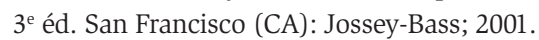
p. $45-66$.

39. Loitz C, Berry TR, Spence JC. 2009 Alberta survey on physical activity: a concise report. Edmonton (AB): Alberta Centre for Active Living; 2009.

40. Trost SG, Owen N, Bauman AE, Sallis JF, Brown W. Correlates of adults' participation in physical activity: review and update. Med Sci Sports Exerc. 2002;34(12):1996-2001.

41. Seefeldt V, Malina RM, Clark MA. Factors affecting levels of physical activity in adults. Sports Med. 2002;32(3):143-68.

42. Ross CE. Walking, exercising, and smoking: Does neighborhood matter? Soc Sci Med. 2000;51(2):265-74.

43. Azevedo MR, Araujo C, Reichert FF, Siqueira FV, da Silva MC, Hallal PC. Gender differences in leisure-time physical activity. Int J Public Health. 2007;52(1):8-15.

44. Barrett JE, Plotnikoff RC, Courneya KS, Raine KD. Physical activity and type 2 diabetes: exploring the role of gender and income. Diabetes Educ. 2007;33(1):128-43.

45. Parsons EM, Betz NE. The relationship of participation in sports and physical activity to body objectification, instrumentality, and locus of control among young women. Psychol Women Q. 2001;25(3):209-22.

46. Stutts WC. Physical activity determinants in adults: perceived benefits, barriers, and self efficacy. AAOHN J. 2002;50(11):499-507.

47. McDermott L. A qualitative assessment of the significance of body perception to women's physical activity experiences: revisiting discussions of physicalities. Sociol Sport J. 2000;17(4):331-63.

48. Thompson AM, Humbert M, Mirwald RL. A longitudinal study of the impact of childhood and adolescent physical activity experiences on adult physical activity perceptions and behaviors. Qual Health Res. 2003;13(3):358-77.
49. Levine JA, McCrady SK, LanninghamFoster LM, Kane PH, Foster RC, Manohar $\mathrm{CU}$. The role of free-living daily walking in human weight gain and obesity. Diabetes. 2008;57(3):548-54.

50. Dishman RK, Sallis JF. Physical activity, fitness, and health: International proceedings and consensus statement. Dans : Bouchard C, Shephard RJ, Stephens T (dir.). Champaign, (IL): Human Kinetics Publishers; 1994. Chapitre 3, Determinants and interventions for physical activity and exercise; p. 214-38.

51. Godin G, Belanger-Gravel A, Nolin B. Mechanism by which BMI influences leisure-time physical activity behavior. Obesity. 2008;16(6):1314-7.

52. Fox KR, Hillsdon M. Physical activity and obesity. Obes Rev. 2007;1:115-21.

53. Lau DC, Douketis JD, Morrison KM, Hramiak IM, Sharma AM, Ur E et collab. 2006 Canadian clinical practice guidelines on the management and prevention of obesity in adults and children. CMAJ. 2007;176(8):117. Sommaire français offert sous forme de PDF (348 Ko) téléchargeable à partir du lien : http://www.cmaj.ca/content /176/8/SF1.full.pdf

54. Physical Activity Advisory Committee. Physical Activity Guidelines Advisory Committee report, 2008. Washington (DC): U.S. Department of Health and Human Services; 2008.

55. Bensimhon DR, Kraus WE, Donahue MP. Obesity and physical activity: a review. Am Heart J. 2006;151(3):598-603.

56. Mortensen LH, Siegler IC, Barefoot JC, Gronbaek M, Sorensen TIA. Prospective associations between sedentary lifestyle and BMI in midlife. Obesity. 2006;14(8):1462-71.

57. Petersen L, Schnohr P, Sorensen TIA Longitudinal study of the long-term relation between physical activity and obesity in adults. Int $\mathrm{J}$ Obes Relat Metab Disord. 2003;28(1):105-12.

58. Giles-Corti B, Donovan RJ. Socioeconomic status differences in recreational physical activity levels and real and perceived access to a supportive physical environment. Prev Med. 2002;35(6):601-11.
59. Booth ML, Macaskill P, Owen N, Oldenburg B, Marcus BH, Bauman A. Population prevalence and correlates of stages of change in physical activity. Health Educ Q. 1993;20(3):431-40.

60. Statistique Canada. Profil d'indicateurs de la santé, estimations annuelles, selon le groupe d'âge et le sexe, Canada, provinces, territoires, régions sociosanitaires (limites de 2007) et groupes de régions homologues, occasionnel. Tableau 105-0501. (Canada) [Internet]. Ottawa (Ont.) : CANSIM (base de données); 2007 [consulté le 18 févr. 2011]. Consultable en ligne à partir de la page : http:// www5.statcan.gc.ca/cansim/a01?lang = fra

61. Statistique Canada. Profil d'indicateurs de la santé, estimations annuelles, selon le groupe d'âge et le sexe, Canada, provinces, territoires, régions sociosanitaires (limites de 2007) et groupes de régions homologues. Tableau 105-0501. (Alberta) [Internet]. Ottawa (Ont) : base de données du CANSIM; 2007 [consulté le 18 mars 2009]. Consultable en ligne à partir de la page : http://www5.statcan. gc.ca/cansim/a01?lang $=$ fra

62. Statistique Canada. Enquête sur la santé dans les collectivités canadiennes (ESCC), questionnaire du cycle 2.1; 2003 [consulté le 17 janv. 2009]. Consultable en ligne à la page : http://www.statcan.gc.ca/concepts /health-sante/cycle2_1/quest-fra.htm

63. Pereira MA, FitzerGerald SJ, Gregg EW, Joswiak ML, Ryan WJ, Suminski RR et collab. A collection of Physical Activity Questionnaires for health-related research. Med Sci Sports Exerc. 1997;29(6 Suppl):S1-205.

64. Centers for Disease Control and Prevention. Adult participation in recommended levels of physical activit-United States, 2001 and 2003. MMWR. 2005;54(47):1208-12.

65. Sjostrom M, Oja P, Hagstromer M, Smith BJ, Bauman A. Health-enhancing physical activity across European Union countries: the Eurobarometer study. J Public Health. 2006;14(5):291-300.

66. Colley RC, Garriguet D, Janssen I, Craig CL, Clarke J, Tremblay MS. Activité physique des adultes au Canada : résultats d'accélérométrie de l'Enquête canadienne sur les mesures de la santé de 2007-2009. Rapport sur la santé. 2011;22(1):15-23. 
67. Rutten A, Abu-Omar K. Prevalence of physical activity in the European Union. Soz.-Praventivmed. 2004;49(4):281-9.

68. Rutten A, Ziemainz H, Schena F, Stahl T, Stiggelbout M, Auweele YV et collab. Using different physical activity measurements in eight European countries. Results of the European Physical Activity Surveillance System (EUPASS) time series survey. Public Health Nutr. 2003;6(4):371-6.

69. Bauman A, Ainsworth BE, Bull F, Craig CL, Hagstromer M, Sallis JF et collab. Progress and pitfalls in the use of the International Physical Activity Questionnaire (IPAQ) for adult physical activity surveillance. J Phys Act Health. 2009;6(1).

70. Friedenreich CM, Cust AE. Physical activity and breast cancer risk: impact of timing, type and dose of activity and population subgroup effects. Brit J Sports Med. 2008;42(8):636-47.

71. Autenrieth C, Schneider A, Doring A, Meisinger C, Herder C, Koenig W et collab. Association between different domains of physical activity and markers of inflammation. Med Sci Sports Exerc. 2009;41(9):1706-13.

72. Ifedi F. La participation sportive au Canada, 2005. Ottawa (Ont.) : Statistique Canada; 2008. PDF (614 Ko) téléchargeable à partir du lien : http://publications.gc.ca/collections /collection_2008/statcan/81-595-M /81-595-MIF2008060.pdf

73. Friedenreich CM, Neilson HK, Lynch BM. State of the epidemiological evidence on physical activity and cancer prevention. Eur J Cancer. 2010;46(14):2593-604.

74. Cerin E, Leslie E, Bauman A, Owen N. Levels of physical activity for colon cancer prevention compared with generic public health recommendations: population prevalence and sociodemographic correlates. Cancer Epidemiol Biomarkers Prev. 2005;14(4):1000-2. 